\title{
The effects of a daily milk supplement on the mental growth and school performance of female primary school children
}

\author{
A. Djazayery ${ }^{1}$, KH. Rahmani ${ }^{2}$, M. Pourshariari ${ }^{2}$, M. A. Habibi ${ }^{2}$ and H. Heydari ${ }^{2}$ \\ ${ }^{1}$ Department of Nutrition and Biochemistry, School of Public Health, Tehran University of Medical Sciences, Tehran, Iran \\ and ${ }^{2}$ National Nutrition and Food Technology Research Institute, Tehran, Iran
}

There is some evidence that regular milk consumption can improve learning ability in children ${ }^{(1)}$. Milk is included in school feeding programmes in some provinces in Iran, but there are no published reports on its potential impact. The objective of this project, approved by the Ethical Committee of the University and conducted according to the guidelines laid down in the Declaration of Helsinki, was to investigate the effects of a regular daily milk supplement on the mental growth and school performance of female primary school children in Tehran.

A group of 469 randomly selected female primary school children (6-10-year-old) with a medium socio-economic status, similar on the average with regard to food consumption and body weights and heights, from four primary schools (two as experimental; $n 232$ and two as control; $n$ 237) were included in the study. Each child received, daily for a period of 3 months, a $250 \mathrm{ml}$ bottle of sterilised homogenised milk and drank it under supervision of the teachers. The Raven Colored Progressive Matrices test and the Wechsler Intelligence Scale (verbal, non-verbal and total IQ), two of the standard psychological tests ${ }^{(2,3)}$, were performed on the subjects initially and at the end of the period, the range of the scores being $<80,80-<90,90-100$ and $>110$. As the table shows, the treatment brought about increases in the proportion of the children with a score of $>110$ in all the tests. The changes in the proportions of those with test scores of $<80$ or of the control groups were statistically non-significant - in most cases negligible. Further analysis of the data also showed that in the experimental group the mean GPA (out of twenty) of 18.1(SD 1.8) in the first quarter increased to $19.4(0.5)$ in the second quarter ( $t$-test, $P<0.05$ ). There was virtually no change $[18.0$ (SD 2.0) -18.0 (SD 2.1)] in the control group.

\begin{tabular}{|c|c|c|c|c|c|c|c|c|c|c|c|c|c|c|c|c|}
\hline & \multicolumn{4}{|c|}{ Raven score } & \multirow{2}{*}{\multicolumn{4}{|c|}{$\begin{array}{c}\text { Wechsler score } \\
\text { Verbal }\end{array}$}} & \multirow{2}{*}{\multicolumn{4}{|c|}{$\frac{\text { Wechsler score }}{\text { Non-verbal }}$}} & \multirow{2}{*}{\multicolumn{4}{|c|}{$\frac{\text { Wechsler score }}{\text { Total IQ }}$}} \\
\hline & & & & & & & & & & & & & & & & \\
\hline & \multicolumn{2}{|c|}{80} & \multicolumn{2}{|c|}{110} & \multicolumn{2}{|c|}{80} & \multicolumn{2}{|c|}{110} & \multicolumn{2}{|c|}{80} & \multicolumn{2}{|c|}{110} & \multicolumn{2}{|c|}{80} & \multicolumn{2}{|c|}{110} \\
\hline & Expt. & $\overline{\text { Cont. }}$ & Expt. & $\overline{\text { Cont. }}$ & Expt. & $\overline{\text { Cont. }}$ & Expt. & $\overline{\text { Cont. }}$ & $\overline{\text { Expt. }}$ & $\overline{\text { Cont. }}$ & Expt. & Cont. & Expt. & Cont. & Expt. & Cont. \\
\hline Initial & 0.0 & 1.7 & 40.1 & 38.1 & 26.6 & 25.1 & 0.4 & 0.5 & 16.4 & 15.1 & 0.8 & 0.8 & 21.4 & 20.0 & 0.4 & $\overline{0.4}$ \\
\hline Final & 0.0 & 1.3 & 65.9 & 45.0 & 13.8 & 25.0 & 11.8 & 0.5 & 8.8 & 14.9 & 28.3 & 0.9 & 8.8 & 25.3 & 14.8 & 8.6 \\
\hline$\chi^{2}, P<$ & NS & NS & 0.05 & NS & NS & NS & 0.05 & NS & NS & NS & 0.05 & NS & NS & NS & 0.05 & NS \\
\hline
\end{tabular}

Expt., experimental; Cont., control; NS, non-significant.

Values are proportions $(\%)$ of children in an experimental or control group.

In conclusion, the findings show that regular daily consumption of $250 \mathrm{ml}$ milk can result in significant improvements in the mental growth and school performance of female primary school children. Thus, it is recommended that milk be included in school feeding programmes in all provinces.

1. Lien do TK, Nhung BT, Khan NC et al. (2009) Asia Pac J Clin Nutr 18, 326-334.

2. Morsanyi K \& Holyoak KJ (2010) Dev Sci 13, 578-587.

3. Brooks BL (2010) Psychol Assess 22, 650-656. 